\title{
IUFOST2006/1023 The role of standardization bodies in the harmonization of analytical methodology in food microbiology
}

\author{
B. Lombard \\ French Agency for Food Safety (AFSSA) LERQAP, Lab. for Study \& Research on Food Quality and on \\ Food Processes, 23 avenue du Général De Gaulle, 94706 Maisons-Alfort, France \\ b.lombard@afssa.fr
}

The need to define reference methods in food microbiology is clear from several points of view. The reference methods being still mainly based on conventional Pasteur microbiology, the result of an analysis is closely linked to the principle of the method used (nature of culture media, time and temperature of incubation of culture media, choice of confirmation tests,oe). This is particularly true for enumeration methods. Moreover, when pathogenic microoganisms are searched, the health of consumers can be directly affected.

Reference methods in food microbiology have been standardized for several years, initially at international level by ISO (International Organization for Standardization) and more recently at European level by CEN (European Committee for Standardization). Namely, two structures are in charge of standardization in that field: Sub-Committee 9 "Microbiology" of the ISO Technical Committee 34 "Food products", and Working Group 6 "Microbial contaminants" of the CEN Technical Committee 275 "Food analysis - horizontal methods". Both structures work in close cooperation to produce Standards either on general aspects (GLP, measurement uncertainty, statistical aspects related to food microbiology,oe), either defining reference methods to detect and/or to enumerate bacteria (and viruses in the future) of interest in food hygiene, either pathogenic or not. Current works on general aspects include the development of a guide on the specific aspects of proficiency testing for food microbiology, as well as a set of Standards on method validation (validation of proprietary methods -revision of EN ISO 16140, in-house method validation, validation of reference methods, method verification). Regarding reference methods, a new Standard is prepared on Enterobacter sakazakii, and a group is dealing with the analysis of samples from the primary production stage (the conditions of use of Standard methods initially developed for food).

The European Commission intends to give a mandate to CEN to validate through inter-laboratory trials all the reference methods standardized by CEN and ISO which are referenced into a recent Regulation on microbiological criteria (EC Regulation 2073/2005). This will increase the scientific validity of these Standard methods. 